\title{
Calcipotriol Plus Betamethasone Dipropionate Aerosol Foam in Patients with Moderate-to-Severe Psoriasis: Sub-Group Analysis of the PSO-ABLE Study
}

\author{
Carle Paul $^{1}$ - Craig Leonardi ${ }^{2}$ Alan Menter ${ }^{3} \cdot$ Kristian Reich $^{4}$. \\ Linda Stein Gold ${ }^{5} \cdot$ Richard B. Warren $^{6} \cdot$ Anders Møller $^{7}$. \\ Mark Lebwohl ${ }^{8}$
}

Published online: 24 February 2017

(c) The Author(s) 2017. This article is published with open access at Springerlink.com

\begin{abstract}
Background Fixed-combination calcipotriol $50 \mu \mathrm{g} / \mathrm{g}$ plus betamethasone $0.5 \mathrm{mg} / \mathrm{g}$ (Cal/BD) aerosol foam is a new topical treatment for psoriasis. Although moderate-to-severe psoriasis is typically treated with systemic/biologic therapies, a topical treatment that is efficacious in these patients may be a significant cost-saving alternative to systemic therapy.

Objective The objective of this study was to assess the response to $\mathrm{Cal} / \mathrm{BD}$ foam and gel in patients with moderate-to-severe psoriasis enrolled in the phase III, 12-week PSO-ABLE study.
\end{abstract}

Mark Lebwohl

lebwohl@aol.com

1 Paul Sabatier University and Larrey Hospital, Toulouse, France

2 Saint Louis University School of Medicine, St. Louis, MO, USA

3 Baylor University Medical Center, Dallas, TX, USA

4 Dermatologikum Hamburg and SCIderm GmbH, Hamburg, Germany

5 Henry Ford Health System, Detroit, MI, USA

6 The Dermatology Centre, Salford Royal NHS Foundation Trust, The University of Manchester, Manchester Academic Health Science Centre, Manchester, UK

7 LEO Pharma A/S, Ballerup, Denmark

8 Department of Dermatology, Icahn School of Medicine at Mount Sinai, 5 E 98th St, New York, NY 10029, USA
Methods Patients eligible for this analysis had moderateto-severe psoriasis, defined by the 'Rule of Tens': body surface area $\geq 10 \%$ or Psoriasis Area and Severity Index (PASI) [excluding head; modified PASI (mPASI)] $>10$ or Dermatology Life-Quality Index $>10$. Endpoints included: proportion of patients achieving mPASI75 or mPASI90; change in body surface area; proportion of patients clear/ almost clear with $a \geq 2$ grade improvement (i.e., treatment success); change in Dermatology Life-Quality Index.

Results Seventy-seven Cal/BD foam patients and 82 gel patients had moderate-to-severe psoriasis. A greater proportion achieved mPASI75 and mPASI90 with Cal/BD foam than gel at weeks 4,8 , and 12 (57.1 vs. 35.4\%; $p=0.006$ and 15.6 vs. $12.2 \%$ at week 12 , respectively); overall reduction in mPASI from baseline to week 12 was $64 \%$ with the foam vs. $51 \%$ with the gel. Overall reduction in body surface area at week 12 was $50 \%$ with the foam and $39 \%$ with the gel. Treatment success rates were higher with the Cal/BD foam than the gel at weeks $1,2,4,8$ ( $p=0.0089$ ), and 12, and a greater proportion of foam patients achieved a Dermatology Life-Quality Index score of $0 / 1$ at weeks 4 ( $p=0.004), 8$, and 12 ( $p=0.001)$.

Conclusion $\mathrm{Cal} / \mathrm{BD}$ foam can be considered as a treatment option in some patients with moderate-to-severe psoriasis who are potential candidates for systemic therapy.

Clinicaltrials.gov identifier NCT02132936. 


\section{Key Points}

A greater proportion of patients achieved modified Psoriasis Area and Severity Index 75 and 90 at weeks 4,8 , and 12 with the calcipotriol $50 \mu \mathrm{g} / \mathrm{g}$ plus betamethasone $0.5 \mathrm{mg} / \mathrm{g}(\mathrm{Cal} / \mathrm{BD})$ foam than the $\mathrm{Cal} / \mathrm{BD}$ gel.

A greater proportion of $\mathrm{Cal} / \mathrm{BD}$ foam patients achieved Dermatology Life-Quality Index 0/1 at weeks 4,8 , and 12 compared with the $\mathrm{Cal} / \mathrm{BD}$ gel patients.

$\mathrm{Cal} / \mathrm{BD}$ aerosol foam may provide an option in some patients who are potential candidates for systemic therapy.

\section{Introduction}

Psoriasis vulgaris is a chronic immune-mediated inflammatory disorder characterized by itchy scaly plaques and thickened skin $[1,2]$. Guidelines for the treatment of psoriasis recommend that mild-to-moderate disease be treated with topical therapies [3-7]. Moderate-to-severe psoriasis is typically treated with systemic and biologic therapies, which have a different risk/benefit profile than topical therapies. Furthermore, the pharmacoeconomic perspective must also be considered when treating psoriasis. As such, a topical treatment that is efficacious in moderate-to-severe patients has the potential to be a significant cost-saving alternative to systemic therapy in some patients.

The efficacy and safety of fixed-combination calcipotriol $50 \mu \mathrm{g} / \mathrm{g}$ (Cal) plus betamethasone $0.5 \mathrm{mg} / \mathrm{g}$ (BD) has been confirmed in long-term studies [8-11], with both the ointment and gel formulations established as first-line treatments for mild-to-moderate psoriasis [12]. An aerosol foam formulation of fixed-combination $\mathrm{Cal} / \mathrm{BD}$ has been developed as a new topical treatment option for psoriasis, with the aim of enhancing adherence and increasing the therapeutic options available. Previous studies with $\mathrm{Cal} / \mathrm{BD}$ aerosol foam have shown greater in vitro drug penetration and a greater anti-psoriatic effect over 4 weeks of treatment than $\mathrm{Cal} / \mathrm{BD}$ ointment and vehicle, with a comparable tolerability profile [13-16]. The phase III PSO-ABLE (LEO90100 in PSOriasis-the effect of prolonged use of calcipotriol And Betamethasone dipropionate combination therapy, a randomized, active- and vehicLE-controlled 12-week trial) study in patients with mild-to-severe psoriasis involving $<30 \%$ body surface area (BSA) demonstrated that Cal/BD aerosol foam had superior efficacy at week 4 compared with $\mathrm{Cal} / \mathrm{BD}$ gel at week 8 [17]; these timepoints are the treatment periods in the approved US Food and Drug Administration prescribing information and the European Medicines Agency summary of product characteristics, and reflect the recommended use of each formulation in clinical practice. This post hoc analysis from PSO-ABLE investigates the efficacy of Cal/BD aerosol foam and gel formulations in the sub-group of patients with moderate-to-severe psoriasis who are candidates for systemic therapy.

\section{Materials and Methods}

\subsection{Study Design}

The PSO-ABLE study was a phase III, prospective, multicenter, investigator-blinded study (NCT02132936) conducted in the UK, USA, and France. The institutional review board or independent ethics committee of all investigational sites approved the protocol and the study was performed in accordance with the Declaration of Helsinki and Good Clinical Practice. Patients were randomized 4:4:1:1 to once-daily Cal/BD aerosol foam, $\mathrm{Cal} /$ $\mathrm{BD}$ gel, foam vehicle, or gel vehicle formulation for up to 12 weeks. The data presented here focus on the active treatment groups rather than the vehicle groups, which were included for blinding purposes only. The full study design details are detailed in Paul et al. [17].

\subsection{Patients}

Patients eligible for inclusion in the PSO-ABLE study were aged $\geq 18$ years with mild-to-severe psoriasis vulgaris according to the 5-point Physician's Global Assessment (PGA) of disease severity, involving 2-30\% BSA (trunk and/or limbs) and a modified (excluding the head, which was not treated) Psoriasis Area and Severity Index (mPASI) of $\geq 2$. For inclusion in this sub-group analysis, a patient was required to have 'moderate-to-severe' psoriasis based on the 'Rule of Tens' [18]: BSA affected $\geq 10 \%$ or mPASI score $>10$ or Dermatology Life Quality Index (DLQI) score $>10$. The full inclusion/exclusion criteria are detailed in Paul et al. [17].

\subsection{Assessments and Endpoints}

The efficacy of Cal/BD aerosol foam and gel was assessed in this moderate-to-severe patient population at weeks 4,8 , and 12 by calculating the proportion of patients achieving a $\geq 75$ or $\geq 90 \%$ reduction in mPASI, the change from baseline in BSA affected by psoriasis, and the proportion of patients who were clear/almost clear of psoriasis with $\mathrm{a} \geq 2$ 
grade improvement according to PGA, defined as 'treatment success'. Patients who achieved treatment success were allowed to discontinue treatment at the investigator's discretion; patients were asked to remain on the study and attend all scheduled visits, but were advised to reinitiate treatment if the psoriasis reappeared on previously treated areas.

To determine the effect of the Cal/BD aerosol foam and gel on quality of life (QoL), patients completed the DLQI questionnaire (range 0-30) at baseline, and weeks 4, 8, and 12. The QoL endpoints included: proportion of patients achieving a DLQI score of 0/1 (i.e., no impact of psoriasis on the patient's life) and the proportion of patients achieving a decrease in DLQI score of $\geq 5$ (i.e., the minimal clinically important difference in DLQI) from baseline to each assessment timepoint. The amount of each product used throughout the treatment period was also assessed.

\subsection{Statistical Analysis}

All statistical analyses were conducted on the full analysis set, which comprised all patients with moderate-to-severe psoriasis. Continuous and ordinal categorical outcomes were compared using the Wilcoxon test, and binary categorical outcomes compared using the Chi square test. The last observation carried forward method was used to impute values for patients with missing mPASI data. An observed case approach was used for all other variables.

\section{Results}

\subsection{Patients}

Overall, 504 patients from 41 centers (France, $n=11$; UK, $n=15$; USA, $n=15$ ) were enrolled; 463 patients were randomized to Cal/BD aerosol foam $(n=185)$, Cal/BD gel $(n=188)$, aerosol foam vehicle $(n=47)$, and gel vehicle $(n=43)$; vehicle groups were included for control purposes only. Seventy-seven Cal/BD aerosol foam patients and $82 \mathrm{Cal} / \mathrm{BD}$ gel patients were classified as having moderate-to-severe psoriasis, as previously defined; baseline demographics and disease characteristics were comparable between these two groups of patients (Table 1).

\section{2 mPASI scores}

The proportion of patients achieving mPASI75 and mPASI90 was generally significantly greater with Cal/BD aerosol foam than Cal/BD gel at weeks 4, 8, and 12 (Fig. 1); the proportions were also greater at week 4 with $\mathrm{Cal} / \mathrm{BD}$ aerosol foam than at week 8 with Cal/BD gel. The overall percentage mean $( \pm$ standard deviation) reduction in $\mathrm{mPASI}$ from baseline to week 12 was $63.8 \pm 40.7$ with the Cal/BD aerosol foam and $50.8 \pm 55.2$ with the $\mathrm{Cal} / \mathrm{BD}$ gel.

\subsection{BSA}

The proportion of BSA affected by psoriasis decreased throughout treatment in both the Cal/BD aerosol foam and $\mathrm{Cal} / \mathrm{BD}$ gel groups; the differences between the Cal/BD aerosol foam and gel were significant at weeks 8 and 12 (Fig. 2). The overall percentage mean ( \pm standard deviation) reduction from baseline to week 12 in BSA affected was $50.2 \pm 43.0 \%$ with the Cal/BD aerosol foam compared with $39.2 \pm 37.7 \%$ for the $\mathrm{Cal} / \mathrm{BD}$ gel.

\subsection{Treatment Success}

Treatment success rates, i.e., patients who were clear/almost clear of psoriasis with a $\geq 2$ grade improvement according to PGA, increased throughout the first 6 weeks, reaching $32.0 \%$ by week 4 in the $\mathrm{Cal} / \mathrm{BD}$ aerosol foam group; these rates continued to increase up to week 12 (Fig. 3). Success rates were higher with Cal/BD aerosol foam than $\mathrm{Cal} / \mathrm{BD}$ gel at each timepoint throughout the study; this difference was significant at week 8 .
Table 1 Patient demographics and disease characteristics at baseline

\begin{tabular}{lll}
\hline & Cal/BD aerosol foam $(n=77)$ & Cal/BD gel $(n=82)$ \\
\hline Male:female ratio, \% & $64: 36$ & $57: 43$ \\
Age, years & $53.2 \pm 12.9$ & $52.1 \pm 14.8$ \\
Body mass index, $\mathrm{kg} / \mathrm{m}^{2}$ & $31.3 \pm 6.0$ & $30.7 \pm 6.3$ \\
Duration of psoriasis, years & $18.4 \pm 13.1$ & $19.6 \pm 15.1$ \\
BSA, \% & $10.9 \pm 6.8$ & $10.4 \pm 6.4$ \\
mPASI score & $10.2 \pm 5.2$ & $8.9 \pm 4.0$ \\
DLQI score & $10.4 \pm 5.7$ & $12.0 \pm 6.4$
\end{tabular}

All data are mean \pm standard deviation

$B S A$ body surface area, $C a l / B D$ calcipotriol $50 \mu \mathrm{g} / \mathrm{g}$ plus betamethasone $0.5 \mathrm{mg} / \mathrm{g}, D L Q I$ Dermatology Life-Quality Index, mPASI modified Psoriasis Area and Severity Index 

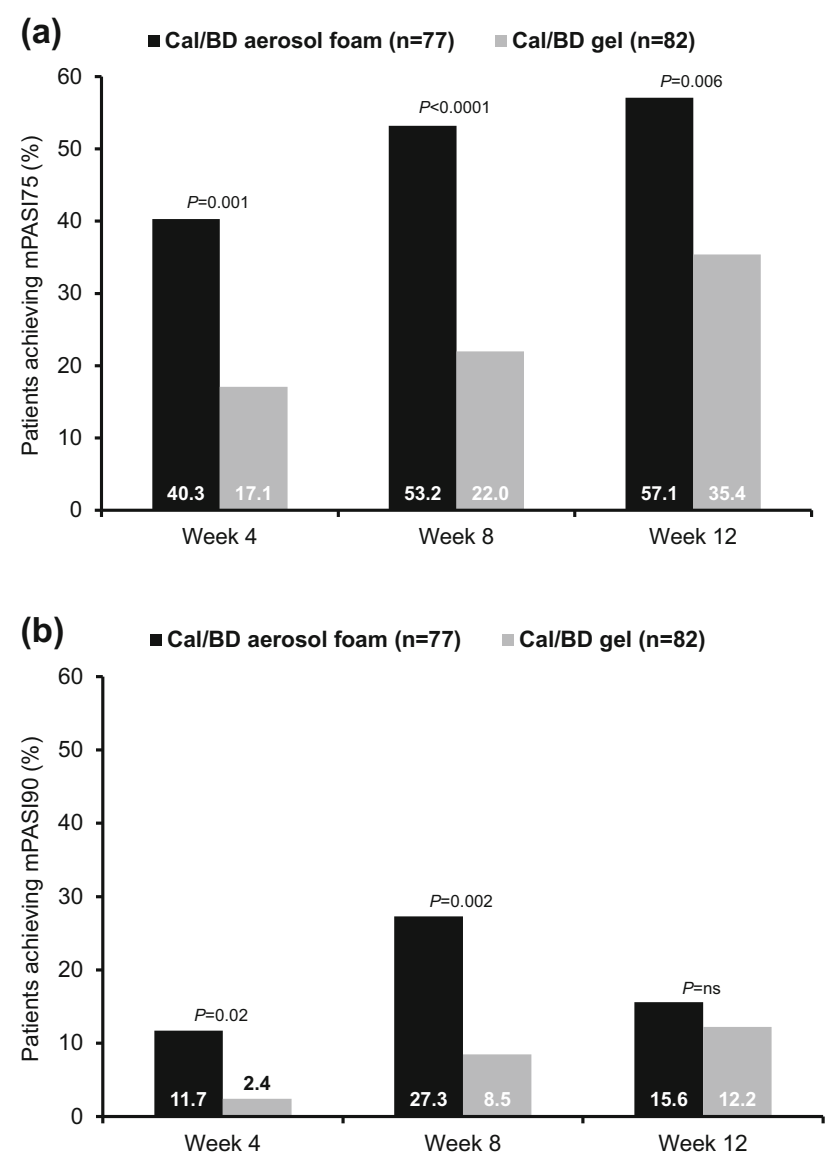

Fig. 1 Proportion of patients with moderate-to-severe psoriasis achieving. a mPASI75 and b mPASI90 during treatment (last observation carried forward). Cal/BD calcipotriol $50 \mu \mathrm{g} / \mathrm{g}$ plus betamethasone $0.5 \mathrm{mg} / \mathrm{g}, \mathrm{mPASI}$ modified Psoriasis Area and Severity Index, $n s$ not significant. $p$ values based on the Chi square test

\subsection{DLQI Scores}

A greater proportion of patients achieved a DLQI score of $0 / 1$ at weeks 4,8 , and 12 with $\mathrm{Cal} / \mathrm{BD}$ aerosol foam than with $\mathrm{Cal} / \mathrm{BD}$ gel; this difference was significant at weeks 4 and 12 (Fig. 4). The proportion of patients achieving a decrease in DLQI of $\geq 5$ with Cal/BD aerosol foam was greater than with $\mathrm{Cal} / \mathrm{BD}$ gel at week 4 (70.3 vs. $56.4 \%)$ but similar at weeks 8 (68.5 vs. $66.2 \%)$ and 12 (62.9 vs. $64.0 \%)$.

\subsection{Amount of Product Used}

The mean amount of Cal/BD aerosol foam used over the 12-week study was $28.0 \pm 20.3 \mathrm{~g} /$ week), compared with $22.6 \pm 18.1 \mathrm{~g} /$ week of $\mathrm{Cal} / \mathrm{BD}$ gel. The greatest usage of $\mathrm{Cal} / \mathrm{BD}$ aerosol foam occurred within the first 6 weeks of the study.

\section{Discussion}

Previous studies have shown that $\mathrm{Cal} / \mathrm{BD}$ aerosol foam has greater efficacy compared with $\mathrm{Cal} / \mathrm{BD}$ ointment and gel [13-16]. With this sub-group analysis from the phase III, 12-week PSO-ABLE study, we have explored the possibility that a proportion of patients with moderate-to-severe psoriasis may be potential candidates for topical treatment and could also benefit from using Cal/BD foam before considering a systemic therapy. This analysis demonstrates that $\mathrm{Cal} / \mathrm{BD}$ aerosol foam is effective in patients with moderate-to-severe psoriasis who are potential candidates
Fig. 2 Change in BSA affected by psoriasis from baseline in moderate-to-severe patients (observed cases). BSA body surface area, $C a l / B D$ calcipotriol $50 \mu \mathrm{g} / \mathrm{g}$ plus betamethasone $0.5 \mathrm{mg} / \mathrm{g}$. $* p=0.02 ;{ }^{* *} p=0.04$; $p$ values based on the Wilcoxon test

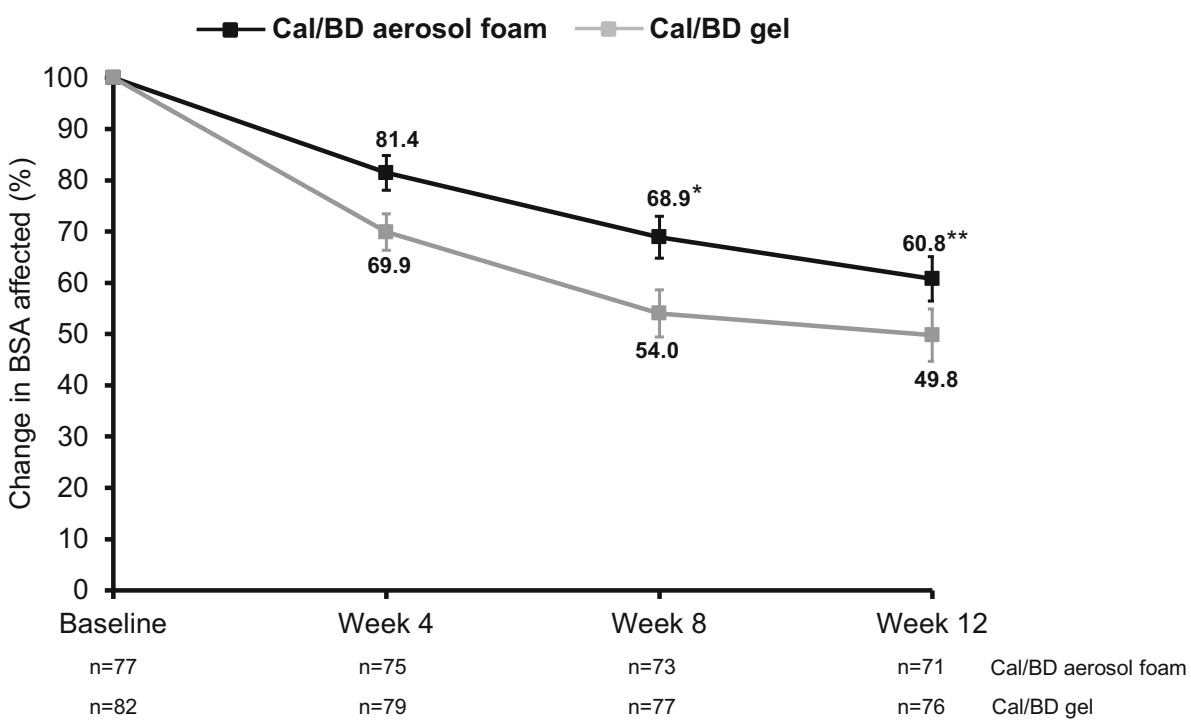


Fig. 3 Proportion of moderateto-severe patients achieving treatment success during treatment (observed cases). $B L$ baseline, $C a l / B D$ calcipotriol $50 \mu \mathrm{g} / \mathrm{g}$ plus betamethasone $0.5 \mathrm{mg} / \mathrm{g}$. $* p=0.0089$; $p$ values based on the Chi square test

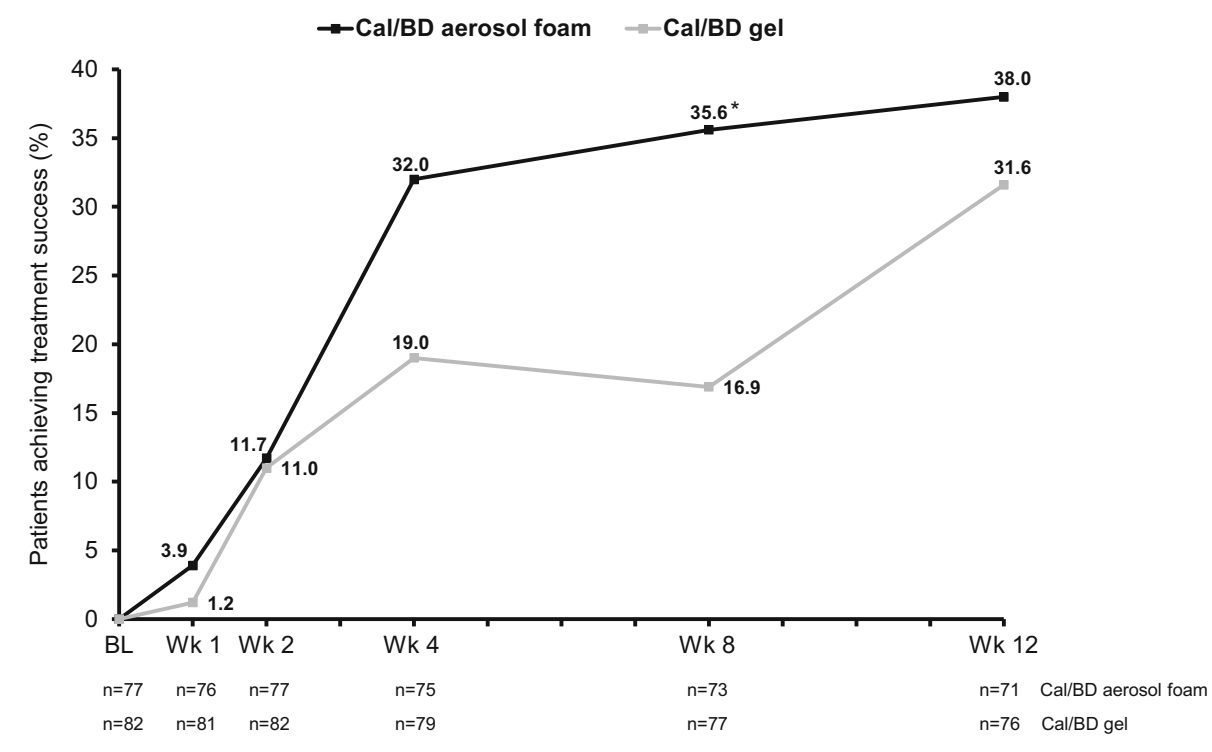

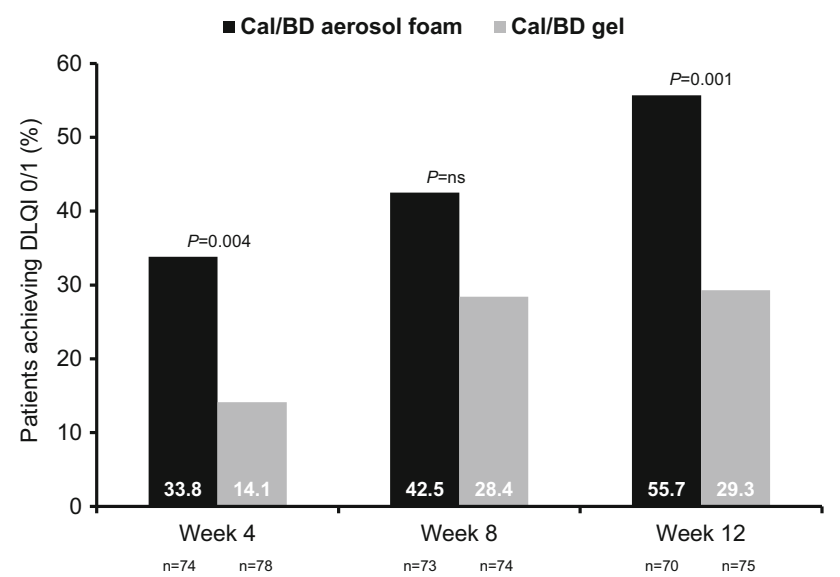

Fig. 4 Proportion of patients achieving a DLQI score of $0 / 1$ (observed cases). Cal/BD calcipotriol $50 \mu \mathrm{g} / \mathrm{g}$ plus betamethasone $0.5 \mathrm{mg} / \mathrm{g}, D L Q I$ Dermatology Life-Quality Index, $n s$ not significant. $p$ values based on the Chi square test

for systemic therapy. More than $50 \%$ of these patients achieve PASI75 with Cal/BD aerosol foam at week 12 . The significantly greater efficacy of $\mathrm{Cal} / \mathrm{BD}$ aerosol foam over $\mathrm{Cal} / \mathrm{BD}$ gel demonstrated in the overall study population [17] is also maintained for up to 12 weeks in patients with moderate-to-severe psoriasis.

By week 12 , almost $60 \%$ of patients receiving $\mathrm{Cal} / \mathrm{BD}$ aerosol foam had achieved mPASI75, which is generally considered the reference standard for treatment responses and efficacy. Almost one-third of $\mathrm{Cal} / \mathrm{BD}$ aerosol foam patients achieved mPASI90 at week 8, although this proportion decreased at week 12 . The proportion of patients with moderate-to-severe psoriasis at baseline who were clear or almost clear was $\sim 30 \%$ by week 4 , with this proportion maintained up to week 12 ; this is comparable to the treatment success rate observed in the overall PSO-ABLE population
[17]. The slight decrease in mPASI90 from weeks 8 to 12 and the steady number of responders from weeks 4 to 12 is likely owing to the study protocol, as patients who achieved treatment success were allowed to discontinue treatment but were required to attend all scheduled visits until study end. These observations also align with the amount of $\mathrm{Cal} / \mathrm{BD}$ aerosol foam used, which was lower from weeks 6 to 12 compared with the first 6 weeks. Of note, $\mathrm{Cal} / \mathrm{BD}$ aerosol foam was effective even though the mean body mass index (BMI) of patients included in this analysis was $>30 \mathrm{~kg} / \mathrm{m}^{2}$. It has been suggested that BMI is a prognostic factor for the response to treatment in psoriasis [19]; indeed, a number of biologic therapies are less effective in patients with a BMI $\geq 30$ compared with a BMI $<30 \mathrm{~kg} / \mathrm{m}^{2}$ [19-23].

By week 4 , one-third of patients with moderate-to-severe psoriasis using Cal/BD aerosol foam had a DLQI score of $0 / 1$, which indicates psoriasis had no effect on patients' QoL; this proportion increased to $>50 \%$ by week 12 . This rapid improvement in QoL, which was maintained throughout the course of treatment, demonstrates that even moderate-to-severe psoriasis had no impact on the daily life of many $\mathrm{Cal} / \mathrm{BD}$ aerosol foam users enrolled in the study. Improvements in patient QoL, in addition to treatment efficacy, is an important treatment objective for prescribers and patients alike. The proportion of patients with moderate-to-severe psoriasis who achieved a reduction in DLQI score of $\geq 5$-the minimal change in which patients identify the improvement as being 'clinically meaningful' [24]—was $70 \%$ at week 4 and decreased slightly to $63 \%$ by week 12 with $\mathrm{Cal} / \mathrm{BD}$ aerosol foam. This implies that factors beyond efficacy impact on the DLQI score and, as discussed previously, may also be a reflection of patients discontinuing treatment as per the study protocol. 
Based upon the criteria for inclusion in this post hoc analysis, the selected population represents the less severe subgroup of moderate-to-severe psoriasis patients. The definition of 'moderate-to-severe' used in this analysis (based on the 'Rule of Tens') differs from that reported in the primary PSO-ABLE article [17], which was based on PGA. While PGA is a static assessment of disease severity, the 'Rule of Tens' is a more holistic assessment of severity and the burden of disease on the patient; this is important because although some patients may not be perceived as having severe psoriasis based on PGA, they may still be candidates for systemic treatment owing to the impact on their QoL. The different definitions used explain the small data differences between these two papers in patients with moderate and severe psoriasis. The definition also differs from that used in studies of systemic therapies, where patients are typically required to have a BSA affected $\geq 10 \%$ and a PASI score $>10$. The mean BSA, mPASI, and DLQI scores in the population included in this analysis were very close to 10 , and therefore, on the threshold for moderate-to-severe psoriasis defined in systemic therapy studies. In addition, as demonstrated in the primary study [17], many of the patients included in this analysis had received prior treatment with systemic therapies. These factors may lead to some bias and should be considered when evaluating these data. In addition, because of the way PASI is calculated, it has relatively poor sensitivity to change in patients with low BSA (e.g., <10\%) [25]. Nevertheless, the efficacy of $\mathrm{Cal} / \mathrm{BD}$ aerosol foam may make it a suitable option for some patients with moderate-to-severe psoriasis who have $<30 \%$ BSA involvement as an alternative to systemic treatments.

\section{Conclusion}

Cal/BD aerosol foam has been shown to be effective in patients with moderate-to-severe psoriasis (as defined by the 'Rule of Tens') and may therefore provide an option for some patients who are potential candidates for systemic therapy.

Acknowledgements Medical writing support was provided by Andrew Jones, Ph.D., from Mudskipper Business Ltd, funded by LEO Pharma. We also thank Dr. Cristina Bulai Livideanu, Paul Sabatier University, Toulouse, France, for her involvement in the study.

\section{Compliance with Ethical Standards}

Funding This study was sponsored by LEO Pharma.

Conflict of interest Carle Paul has been an investigator and consultant for AbbVie, Amgen, Boehringer Ingelheim, Celgene, GSK, Janssen Cilag, LEO Pharma, Lilly, Novartis, and Pfizer. Craig Leonardi has been a consultant for LEO Pharma, AbbVie, Amgen,
Dermira, Lilly, Janssen, Sandoz, UCB, and Pfizer, received honoraria from AbbVie, Amgen, Dermira, Lilly, Sandoz, UCB, Pfizer, Celgene, and Novartis, and has participated in speakers' bureaus for AbbVie and Celgene. Alan Menter reports grants and honoraria from AbbVie, Allergan, Amgen, Boehringer Ingelheim, Genentech, Janssen Biotech, LEO Pharma, Novartis, Pfizer, and Syntrix, honoraria from Convoy Therapeutics, Lilly, Vitae, Wyeth, and Xenoport, and grants from Celgene, Merck, and Symbio/Maruho. Kristian Reich reports receiving grants and/or honoraria from AbbVie, Amgen, Biogen, Boehringer Ingelheim, Celgene, Covagen, Forward Pharma, GSK, Janssen-Cilag, Lilly, LEO Pharma, Medac, Novartis, Pfizer, Regeneron, Takeda, UCB Pharma, and Xenoport. Linda Stein Gold has been an investigator and advisor for LEO Pharma. Richard B. Warren has acted as a consultant and or speaker for Amgen, AbbVie, Janssen, LEO Pharma, Lilly, Novartis, and Pfizer and has received grant support from AbbVie, Janssen, Novartis, and Pfizer. Anders Møller is an employee of LEO Pharma. Mark Lebwohl is an employee of the Mount Sinai Medical Center, which receives research funds from AbGenomics, Amgen, Anacor, Boehringer Ingelheim, Celgene, Ferndale, Lilly, Janssen Biotech, Kadmon, LEO Pharma, Medimmune, Novartis, Pfizer, Sun Pharmaceuticals, and Valeant.

Ethics approval The institutional review board or independent ethics committee of all investigational sites approved the protocol and the study was performed in accordance with the Declaration of Helsinki and Good Clinical Practice. All patients provided written informed consent.

Open Access This article is distributed under the terms of the Creative Commons Attribution-NonCommercial 4.0 International License (http://creativecommons.org/licenses/by-nc/4.0/), which permits any noncommercial use, distribution, and reproduction in any medium, provided you give appropriate credit to the original author(s) and the source, provide a link to the Creative Commons license, and indicate if changes were made.

\section{References}

1. Menter A, Gottlieb A, Feldman SR, et al. Guidelines of care for the management of psoriasis and psoriatic arthritis. Section 1: overview of psoriasis and guidelines of care for the treatment of psoriasis with biologics. J Am Acad Dermatol. 2008;58:826-50.

2. Schön MP, Boehncke W-H. Psoriasis. N Engl J Med. 2005;352:1899-912.

3. Menter A, Korman NJ, Elmets CA, et al. Guidelines of care for the management of psoriasis and psoriatic arthritis. Section 3: guidelines of care for the management and treatment of psoriasis with topical therapies. J Am Acad Dermatol. 2009;60:643-59.

4. Nast A, Boehncke WH, Mrowietz U, et al. German S3-guidelines on the treatment of psoriasis vulgaris (short version). Arch Dermatol Res. 2012;304:87-113.

5. Samarasekera E, Sawyer L, Parnham J, et al. Assessment and management of psoriasis: summary of NICE guidance. BMJ. 2012;345:e6712.

6. Paul C, Gallini A, Archier E, et al. Evidence-based recommendations on topical treatment and phototherapy of psoriasis: systematic review and expert opinion of a panel of dermatologists. J Eur Acad Dermatol Venereol. 2012;26(Suppl. 3):1-10.

7. Devaux S, Castela A, Archier E, et al. Topical vitamin D analogues alone or in association with topical steroids for psoriasis: a systematic review. J Eur Acad Dermatol Venereol. 2012;26(Suppl. 3):52-60.

8. Kragballe K, Austad J, Barnes L, et al. Efficacy results of a 52-week, randomised, double-blind, safety study of a 
calcipotriol/betamethasone dipropionate two-compound product (Daivobet ${ }^{\circledR} /$ Dovobet $^{\circledR} /$ Taclonex $^{\circledR}$ ) in the treatment of psoriasis vulgaris. Dermatology. 2006;213:319-26.

9. Kragballe K, Austad J, Barnes L, et al. A 52-week randomized safety study of a calcipotriol/betamethasone dipropionate twocompound product (Dovobet ${ }^{\circledR} /$ Daivobet $^{\circledR} /$ Taclonex $^{\circledR}$ ) in the treatment of psoriasis vulgaris. $\mathrm{Br} \mathrm{J}$ Dermatol. 2006;154:1155-60.

10. Lambert J, Hol CW, Vink J. Real-life effectiveness of once-daily calcipotriol and betamethasone dipropionate gel vs. ointment formulations in psoriasis vulgaris: final analysis of the 52-week PRO-long study. J Eur Acad Dermatol Venereol. 2015;29:2349-55.

11. Luger TA, Cambazard F, Larsen FG, et al. A study of the safety and efficacy of calcipotriol and betamethasone dipropionate scalp formulation in the long-term management of scalp psoriasis. Dermatology. 2008;217:321-8.

12. Laws PM, Young HS. Topical treatment of psoriasis. Expert Opin Pharmacother. 2010;11:1999-2009.

13. HollesenBasse L, Olesen M, Lacour JP, et al. Enhanced in vitro skin penetration and antipsoriatic effect of fixed combination calcipotriol plus betamethasone dipropionate in an innovative foam vehicle. J Investig Dermatol. 2014;134:S33 (abstract no. 192).

14. Koo J, Tyring S, Werschler WP, et al. Superior efficacy of calcipotriene and betamethasone dipropionate aerosol foam versus ointment in patients with psoriasis vulgaris: a randomized phase II study. J Dermatol Treat. 2016;27:120-7.

15. Leonardi C, Bagel J, Yamauchi P, et al. Efficacy and safety of calcipotriene plus betamethasone dipropionate aerosol foam in patients with psoriasis vulgaris: a randomized phase III study (PSO-FAST). J Drugs Dermatol. 2015;14:1468-77.

16. Queille-Roussel C, Olesen M, Villumsen J, et al. Efficacy of an innovative aerosol foam formulation of fixed combination calcipotriol plus betamethasone dipropionate in patients with psoriasis vulgaris. Clin Drug Investig. 2015;35:239-45.

17. Paul C, Stein Gold L, Cambazard F, et al. Calcipotriol plus betamethasone dipropionate aerosol foam provides superior efficacy versus gel in patients with psoriasis vulgaris: randomized, controlled PSO-ABLE study. J Eur Acad Dermatol Venereol. 2017;31(1):119-26.

18. Finlay AY. Current severe psoriasis and the Rule of Tens. Br J Dermatol. 2005;152:861-7.

19. Naldi L, Addis A, Chimenti S, et al. Impact of body mass index and obesity on clinical response to systemic treatment for psoriasis: evidence from the Psocare project. Dermatology. 2008;217:365-73.

20. Cassano N, Galluccio A, De Simone C, et al. Influence of body mass index, comorbidities and prior systemic therapies on the response of psoriasis to adalimumab: an exploratory analysis from the APHRODITE data. J Biol Regul Homeost Agents. 2008;22:233-7.

21. Umezawa Y, Saeki H, Nakagawa H. Some clinical factors affecting quality of the response to ustekinumab for psoriasis. J Dermatol. 2014;41:690-6.

22. Yanaba K, Umezawa $Y$, Ito $T$, et al. Impact of obesity on the efficacy of ustekinumab in Japanese patients with psoriasis: a retrospective cohort study of 111 patients. Arch Dermatol Res. 2014;306:921-5.

23. Puig L. Obesity and psoriasis: body weight and body mass index influence the response to biological treatment. J Eur Acad Dermatol Venereol. 2011;25:1007-11.

24. Shikiar R, Willian MK, Okun MM, et al. The validity and responsiveness of three quality of life measures in the assessment of psoriasis patients: results of a phase II study. Health Qual Life Outcomes. 2006;4:71.

25. Feldman SR, Krueger GG. Psoriasis assessment tools in clinical trials. Ann Rheum Dis. 2005;64(Suppl. 2):ii65-8. 